\title{
Kinetic theory of the heat conductivity in the crust of a magnetized neutron star
}

\section{G. S. Bisnovatyi-Kogan*}

Space Research Institute, Russian Academy of Sciences,

Profsoyuznaya 84/32, Moscow 117997, Russia

and

National Research Nuclear University MEPhI

(Moscow Engineering Physics Institute), Kashirskoe Shosse 31, Moscow 115409, Russia

E-mail: gkoganeiki.rssi.ru

\section{V. Glushikhina}

Space Research Institute, Russian Academy of Sciences,

Profsoyuznaya 84/32, Moscow 117997, Russia

E-mail: m.glushikhina@iki.rssi.ru

\begin{abstract}
The solution of Boltzmann equation for plasma in magnetic field, with arbitrarily degenerate electrons and non-degenerate nuclei, is obtained by Chapman-Enskog method. Fully ionized plasma is considered. The tensor of the heat conductivity coefficients in non-quantized magnetic field is calculated. For strongly degenerate plasma the asymptotic analytic formulas are obtained. The Lorentz approximation, with neglecting of electron-electron encounters, is asymptotically exact for strongly degenerate plasma. For strongly degenerate electrons we obtain an asymptotically exact analytical solution for the heat conductivity tensor in presence of a magnetic field. This solution has considerably more complicated dependence on the magnetic field than those in previous publications, and gives several times smaller relative value of a thermal conductivity across the magnetic field at $\omega \tau \gtrsim 0.8$.
\end{abstract}

XII Multifrequency Behaviour of High Energy Cosmic Sources Workshop

12-17 June 2017

Mondello (Palermo), Italy

\footnotetext{
*Speaker.
} 


\section{Introduction}

X-ray observations of thermal emission show periodic variabilities in single neutron stars[23], indicating to the anisotropic temperature distribution. Such kind of a single neutron star variability was predicted in [4] in 1976 year. A periodic X-ray variability of a single neutron star is presented in Fig.1 from [10].

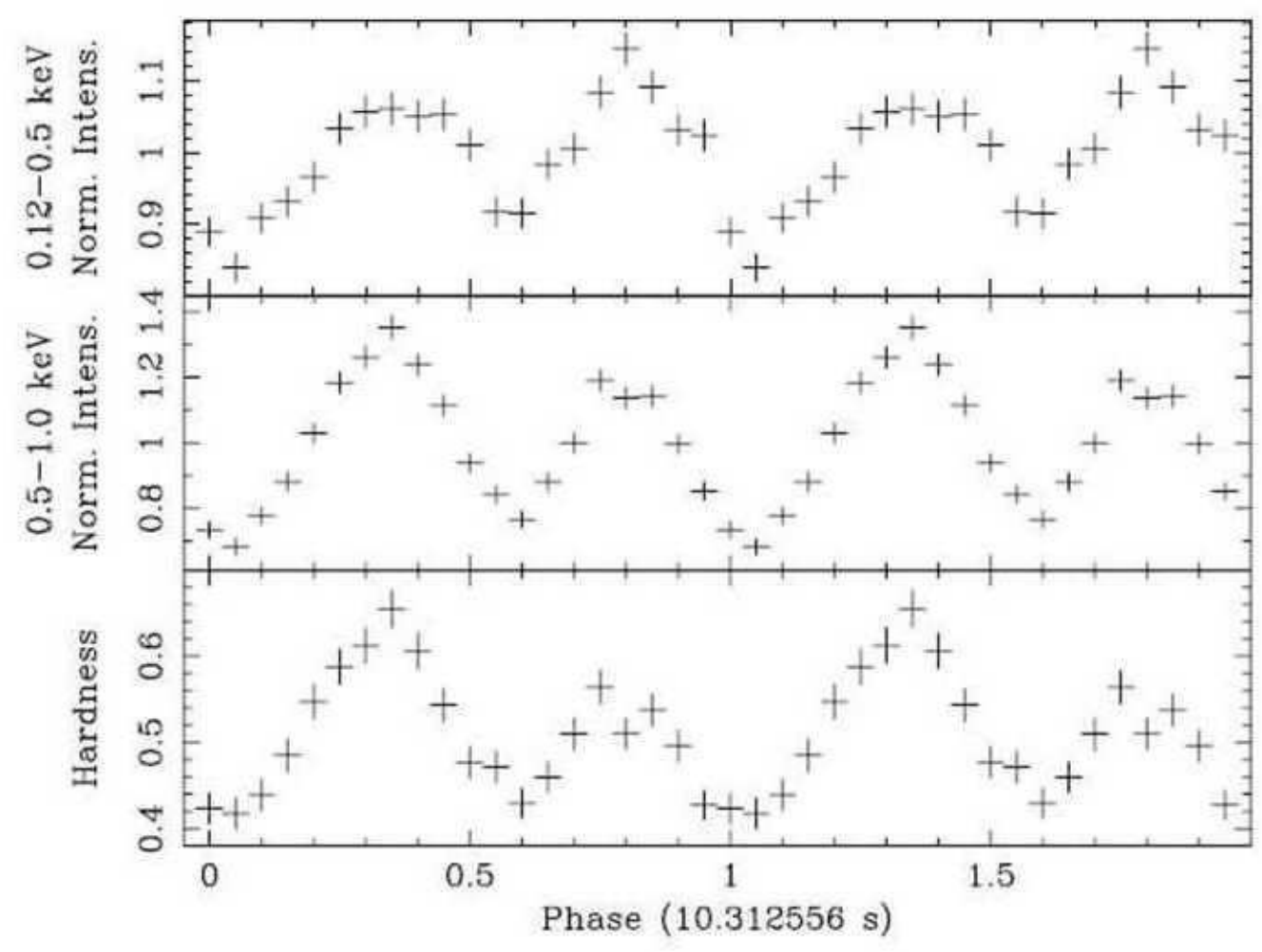

Figure 1: Pulse profile of RBS1223 in the $0.012-0.5 \mathrm{keV}$ (soft) and $0.5-1 \mathrm{keV}$ (hard) energy bands, together with the ratio hard/soft obtained from the EPIC-pn data of the Jan 2003 XMM observations, from [10].

The list of 7 known single neutron star, named in the literature as "magnifucent seven", with some of their properties, is presented in Fig.2, see [19].

Heat transfer in the envelopes of NS plays crucial role in many aspects of evolution of these stars. Thermal conductivity is the basic quantity needed for calculating the relationship between the internal temperature of a neutron star and its effective surface temperature. To calculate thermal conductivity we should know the transport properties of a dense matter where electrons are degenerate, and form a nearly ideal Fermi-gas [22]. The ions are usually treated as non-degenerate. They may be in a gaseous state, may form a Coulomb liquid or a Coulomb crystal [16]. Under such conditions, electrons are the most important heat carriers, and the thermal conductivity is determined by electron motion. The magnetic field limits the motion of electrons in directions perpendicular to the field lines and, since they are the main carriers of the heat transport, the thermal conductivity in these directions is suppressed, while remaining unaffected along the field lines. The conductivity of 


\begin{tabular}{|l|l|l|l|l|}
\hline Source, RX J & Spin Periods, s & Amplitude/2 & Temperature, eV & $\begin{array}{c}\text { Absorption } \\
\text { line energy, eV }\end{array}$ \\
\hline $1856.5-3754$ & 7.06 & $1.5 \%$ & $60-62$ & no \\
\hline $0720.4-3125$ & 8.39 & $11 \%$ & $85-87$ & 270 \\
\hline $\begin{array}{l}1605.3+3249 \\
\text { (RBS } 1556)\end{array}$ & $? ? ?$ & - & $93-96$ & 450 \\
\hline $\begin{array}{l}1308.6+2127 \\
\text { (RBS 1223) }\end{array}$ & 10.31 & $18 \%$ & 102 & 300 \\
\hline $\begin{array}{l}2143.0+0654 \\
\text { (RBS 1774) }\end{array}$ & 9.44 & $4 \%$ & $102-104$ & 700 \\
\hline $0806.4-4123$ & 11.37 & $6 \%$ & 92 & 460 \\
\hline $0420.0-5022$ & 3.45 & $13 \%$ & 45 & 330 \\
\hline
\end{tabular}

Figure 2: The list of single neutron star X-ray sources (magnificent seven) with their physical characteristics, from [19]

electrons in NS and white dwarfs in presence of a magnetic field was studied in $[8,18]$. The ratio between thermal conductivity along and across magnetic field lines considered in [8] was taken as

$$
\frac{\lambda_{\perp}}{\lambda_{\|}}=\frac{1}{1+(\omega \tau)^{2}} .
$$

and was used also in [18]. Here $\omega$ is electron cyclotron frequency, $\tau$ is the time between collisions. The influence of the magnetic field on the electron heat conductivity in the form (1.1) was used in subsequent papers, see $[11,21]$. Classical methods of kinetic gas theory were developed by Maxwell, Boltzmann, Gilbert, Enskog and Chapman. These methods are presented in the monograph of Chapman and Cowling [7]. Here we find an analytic solution for the heat conductivity tensor of strongly degenerate electrons in a magnetic field, in the Lorentz approximation, which is asymptotically exact in this case, showing a more complicated dependence on the magnetic field strength than (1.1). The kinetic coefficients in the non-degenerate plasma, with and without magnetic field had been calculated in [14, 15, 2, 1], using Chapmen-Enskog expansion method. Coefficients of the heat conductivity tensor in a degenerate stellar cores were calculated in Lorentz approximation for a hydrogen plasma in [20]. A non-relativistic calculation, based on the quantum Lenard-Balescu transport equation for the thermal and electrical conductivities of plasma of highly degenerate, weakly coupled electrons and nondegenerate, weakly coupled ions was performaed in [12].

Here we present the solution of the Boltzmann equation for strongly degenerate electrons in non-quantized magnetic field, described in [3]. For strongly degenerate electrons we obtain an asymptotically exact analytical solution for the heat conductivity tensor in presence of a magnetic field. This solution has considerably more complicated dependence on the magnetic field than those in previous publications, and gives several times smaller relative value of a thermal conductivity 
across the magnetic field.

\section{Boltzmann equations and transfer equations}

We use a Boltzmann equation for electrons, in a magnetic field, with an allowance of arbitrary degeneracy, and assuming them as non-relativistic. We consider the electron gas in a crystal lattice of heavy nuclei, and take into account the interaction of the electrons with a nondegenerate nuclei and with one another. The nuclear component of the matter in the crust is in a crystal state, and therefore the isotropic part of the distribution function $f_{N 0}$ may differ from the Maxwellian distribution. If the mass of the nucleus $m_{N}$ is much greater, than the electron mass $m_{e}$, then to the terms $\sim m_{e} / m_{N}$ the details of the distribution function $f_{N 0}$ are unimportant, and the calculations can be made for arbitrary $f_{N 0}$.

Boltzmann equation, which describes the time variation of the electron distribution function $f$ in presence of the electric and magnetic fields is written as $[2,1]$

$$
\frac{\partial f}{\partial t}+c_{i} \frac{\partial f}{\partial r_{i}}-\frac{e}{m_{e}}\left(E_{i}+\frac{1}{c} \varepsilon_{i k l} c_{k} B_{l}\right) \frac{\partial f}{\partial c_{i}}+J=0 .
$$

Here $(-e), m_{e}$ are the charge (negative) and the mass of the electron, $E_{i}, B_{i}$ are the strength of the electric field, and magnetic induction, $\varepsilon_{i k l}$ is the totally antisymmetric Levi-Civita tensor, $c$ is the speed of the light, $c_{i}$ is a velocity vector in the laboratory frame, $r_{i}$ is a particle radius-vector in the laboratory frame. The collision integral $J$ for arbitrary degenerate electrons, from $[7,17]$, is written in the form,

$$
\begin{array}{r}
J=J_{e e}+J_{e N}=R \int\left[f^{\prime} f_{1}^{\prime}(1-f)\right. \\
\left.+\left(1-f_{1}\right)-f f_{1}\left(1-f^{\prime}\right)\left(1-f_{1}^{\prime}\right)\right] \times g_{e e} b d b d \varepsilon d c_{1 i}+ \\
+\int\left[f^{\prime} f_{N}^{\prime}(1-f)-f f_{N}\left(1-f^{\prime}\right)\right] \times g_{e N} b d b d \varepsilon d c_{N i} .
\end{array}
$$

Here, the impact parameter $b$, and $\varepsilon$ are geometrical parameters of particle collisions with relative velocities $g_{e e}, g_{e N}, R=\frac{2 m_{e}^{3}}{h^{3}}$. The integration in electron part of the collision integral in (2.2) is performed over the phase space of the incoming particles $\left(d c_{1 i}\right)$, and the physical space of their arrival $(b d b d \varepsilon)$ [7]. The velocity functions after collision are marked with touches.

The Boltzmann equation for electrons with a binary collision integral (2.2) may be applied in conditions, when the electron gas is considered as ideal, i.e. the kinetic energy of the electrons is much larger than the energy of electrostatic interactions. In the neutron stars and white dwarfs we have a plasma at very large density, when it is important to take into account the electrons degeneracy. It is known from the statistical physics, that a gas of strongly degenerate electrons becomes ideal, because large Fermi energy substitutes here the thermal energy [13]. Therefore the calculations in this paper are applied to the high density plasma with degenerate electrons. Detailed discussion of the applicability of a binary collision integral (2.2), and its modifications for high density non-degenerate gases may be found in [7]

The collision integral similar to $J_{e e}$ from (2.2) for strongly degenerate neutrons in nuclear matter was written in [17], see also [5]. It was found that that in the presence of non-degenerate heavy nuclei and strongly degenerate neutron, the input of collisions between them in the heat 
transfer and diffusion coefficients is negligibly small, in comparison with neutron-nuclei collisions. The same situation we have for the strongly degenerate electrons. Therefore for strongly degenerate electrons the Lorentz approximation, with account of collisions between light and heavy particles only, is asymptotically exact. So for our consideration we can neglect $J_{e e}$ in comparison with $J_{e N}$, and we can equate $J=J_{e N}$ in the (2.2).

Lets introduce the thermal velocity of electrons, $v_{i}=c_{i}-c_{0 i}$, where $c_{0 i}$ is the mass-average velocity. So we can write the Boltzmann equation with respect to the thermal velocity in the form [1]

$$
\begin{array}{r}
\frac{d f}{d t}+v_{i} \frac{\partial f}{\partial r_{i}}-\left[\frac{e}{m_{e}}\left(E_{i}+\frac{1}{c} \varepsilon_{i k l} v_{k} B_{l}\right)+\frac{d c_{0 i}}{d t}\right] \frac{\partial f}{\partial v_{i}} \\
-\frac{e}{m_{e} c} \varepsilon_{i k l} v_{k} B_{l} \frac{\partial f}{\partial v_{i}}-\frac{\partial f}{\partial v_{i}} v_{k} \frac{\partial c_{0 i}}{\partial r_{k}}+J=0 .
\end{array}
$$

The transfer equations for the electron concentration, total momentum, and electron energy, in the two-component mixture of electrons and nuclei, can be obtained in a usual manner from the Boltzmann equation in a quasi-neutral plasma $[7,15,2,1]$.

\section{Heat conductivity of strongly degenerate electrons in presence of magnetic field: Lorentz approximation}

The Boltzmann equation can be solved by Chapmen-Enskog method of successive approximation [7]. This method is used here for conditions, when distribution functions are close to their values in thermodynamic equilibrium, and deviations are considered in a linear approximation. Equation for second order deviation from the equilibrium distribution function had been derived in [6] for a simple gas, see also [7]. The complexity of this equation, and rather narrow region where second order corrections could be important, strongly restricted the application of this approach.

The zeroth approximation to the electron distribution function is a Fermi-Dirac distribution, which is found by equating to zero of the collision integral $J_{e e}$ from (2.2)

$$
f_{0}=\left[1+\exp \left(\frac{m_{e} v^{2}-2 \mu}{2 k T}\right)\right]^{-1}, \quad R \int f_{0} d v_{i}=n_{e} .
$$

Here, $\mu$ is a chemical potential of electrons, $k$ is Boltzmann's constant, $T$ is the temperature. The nuclear distribution function in the zeroth approximation $f_{N 0}$ is assumed to be isotropic with respect to the velocities and to depend on the local thermodynamic parameters.

In the first approximation, we seek for the function $f$ in the form: $f=f_{0}\left[1+\chi\left(1-f_{0}\right)\right]$.

The function $\chi$ admits representation of the solution in the form:

$$
\begin{gathered}
\chi=-A_{i} \frac{\partial \ln T}{\partial r_{i}}-n_{e} D_{i} d_{i} \frac{G_{5 / 2}}{G_{3 / 2}} \\
d_{i}=\frac{\rho_{N}}{\rho} \frac{\partial \ln P_{e}}{\partial r_{i}}-\frac{\rho_{e}}{P_{e}} \frac{1}{\rho} \frac{\partial P_{N}}{\partial r_{i}}+\frac{e}{k T}\left(E_{i}+\frac{1}{c} \varepsilon_{i k l} c_{0 k} B_{l}\right) .
\end{gathered}
$$


The plasma is supposed to be quasineutral with a zero charge density. The functions $A_{i}$ and $D_{i}$ determine the heat transfer and diffusion. Substituting (3.2) in the equation for $\chi$ we obtain equations for $A_{i}, D_{i}$ [7]. It was shown in [2],[1], that in presence of a magnetic field $B_{i}$, the polar vector $A_{i}$ (and similarly $D_{i}$ ) may be searched for in the form:

$$
A_{i}=A^{(1)} v_{i}+A^{(2)} \varepsilon_{i j k} v_{j} B_{k}+A^{(3)} B_{i}\left(v_{j} B_{j}\right),
$$

Introducing a function: $\quad \xi=A^{(1)}+i B A^{(2)}$, and dimensionless velocity: $u_{i}=\sqrt{\frac{m_{e}}{2 k T}} v_{i}$, we obtain the system for $\xi$ as

$$
\begin{gathered}
f_{0}\left(1-f_{0}\right)\left(u^{2}-\frac{5 G_{5 / 2}}{2 G_{3 / 2}}\right)=-i B f_{0}\left(1-f_{0}\right) \frac{e \xi}{m_{e} c} u_{i} \\
+\int f_{0} f_{N 0}\left(1-f_{0}^{\prime}\right)\left(\xi u_{i}-\xi^{\prime} u_{i}^{\prime}\right) g_{e N} b d b d \varepsilon d c_{N i} .
\end{gathered}
$$

Using relations $f_{0}^{\prime}=f_{0}, \xi^{\prime}=\xi, u_{i}^{\prime}=u_{i} \cos \theta$, and making integration over $d c_{N i}$, the equation for the function $\xi$ may be written in the form:

$$
\begin{array}{r}
f_{0}\left(1-f_{0}\right)\left(u^{2}-\frac{5 G_{5 / 2}}{2 G_{3 / 2}}\right)=-i B f_{0}\left(1-f_{0}\right) \frac{e \xi}{m_{e} c} u_{i} \\
+f_{0}\left(1-f_{0}\right) n_{N} \xi \int(1-\cos \theta) g_{e N} b d b d \varepsilon
\end{array}
$$

The function $\xi$ is defined by expression

$$
\xi=\frac{u^{2}-\frac{5}{2} \frac{G_{5 / 2}}{G_{3 / 2}}}{2 \pi n_{N} \int_{0}^{\infty}(1-\cos \theta) g b d b-i \omega} .
$$

We obtain in Lorenz approximation, with $g_{12}=v$,

$$
\int_{0}^{\infty}(1-\cos \theta) g b d b=2 \frac{e^{4} Z^{2}}{m_{e}^{2} \nu^{3}} \Lambda, \quad \xi=\frac{u^{2}-\frac{5}{2} \frac{G_{5 / 2}}{G_{3 / 2}}}{4 \pi n_{N}\left(\frac{m_{e}}{2 k T}\right)^{3 / 2} \frac{e^{4} Z^{2}}{m_{e}^{2} u^{3}} \Lambda-i \omega} .
$$

According to the complex form of $\xi$ we have:

$$
\begin{gathered}
A^{(1)}=\frac{\left(u^{2}-\frac{5}{2} \frac{G_{5 / 2}}{G_{3 / 2}}\right) 4 \pi n_{N}\left(\frac{m_{e}}{2 k T}\right)^{3 / 2} \frac{e^{4} Z^{2}}{m_{e}^{2} u^{3}} \Lambda}{\left[4 \pi n_{N}\left(\frac{m_{e}}{2 k T}\right)^{3 / 2} \frac{e^{4} Z^{2}}{m_{e}^{2} u^{3}} \Lambda\right]^{2}+\omega^{2}}, \\
A^{(2)=} \frac{\omega}{B} \frac{u^{2}-\frac{5}{2} \frac{G_{5 / 2}}{G_{3 / 2}}}{\left[4 \pi n_{N}\left(\frac{m_{e}}{2 k T}\right)^{3 / 2} \frac{e^{4} Z^{2}}{m_{e}^{2} u^{3}} \Lambda\right]^{2}+\omega^{2}} . \\
A^{(3)}=A^{(1)}(B=0)-A^{(1)} .
\end{gathered}
$$

The expression for the heat flux is written as: 


$$
\begin{array}{r}
q_{i}=-\frac{2 \pi}{3} \frac{m_{e}^{4}}{h^{3} T}\left(\frac{2 k T}{m_{e}}\right)^{7 / 2}\left[\delta_{i j} \int_{0}^{\infty} f_{0}\left(1-f_{0}\right) A^{(1)} x^{5 / 2} d x\right. \\
\left.-\varepsilon_{i j k} B_{k} \int_{0}^{\infty} f_{0}\left(1-f_{0}\right) A^{(2)} x^{5 / 2} d x+B_{i} B_{j} \int_{0}^{\infty} f_{0}\left(1-f_{0}\right) A^{(3)} x^{5 / 2} d x\right] \frac{\partial T}{\partial x_{j}} \\
=-\lambda^{(1)} \frac{\partial T}{\partial x_{i}}-\varepsilon_{i j k} B_{k} \lambda^{(2)} \frac{\partial T}{\partial x_{j}}-B_{i} B_{j} \lambda^{(3)} \frac{\partial T}{\partial x_{j}} \\
=q_{i}^{(1)}+q_{i}^{(2)}+q_{i}^{(3)}, \quad x=u^{2} .
\end{array}
$$

For strongly degenerate electrons at $x_{0} \gg 1$ the integrals in (3.11) with $A^{(1)}, A^{(2)} A^{(3)}$ from (3.9)(3.10) are expressed analytically, using expansion formula [13]

$$
\int_{0}^{\infty} \frac{f(x) d x}{e^{x-x_{0}}+1}=\int_{0}^{x_{0}} f(x) d x+\frac{\pi^{2}}{6} f^{\prime}\left(x_{0}\right)+\ldots
$$

The average frequency of electron-ion collisions $v_{e i}$ is written in [9] in the form

$$
v_{e i}=\frac{4}{3} \sqrt{\frac{2 \pi}{m_{e}}} \frac{Z^{2} e^{4} n_{N} \Lambda}{(k T)^{3 / 2} G_{3 / 2}} \frac{1}{1+e^{-x_{0}}} .
$$

For strongly degenerate electrons it is expressed as

$$
v_{e i}=\frac{32 \pi^{2}}{3} m_{e} \frac{Z^{2} \mathrm{e}^{4} \Lambda n_{N}}{h^{3} n_{\mathrm{e}}} .
$$

Using (3.9), and writing the formula using $\tau_{d}$ as an inverse value of $v_{e i}$ from (3.17), we write the heat conductivity coefficients in the form

$$
\begin{gathered}
\lambda^{(1)}=\frac{5 \pi^{2}}{6} \frac{k^{2} T n_{e}}{m_{e}} \tau_{d}\left\{\frac{1}{1+\omega^{2} \tau_{d}^{2}}-\frac{6}{5} \frac{\omega^{2} \tau_{d}^{2}}{\left(1+\omega^{2} \tau_{d}^{2}\right)^{2}}-\left.\frac{\pi^{2}}{10}\left[\frac{1}{1+\omega^{2} \tau_{d}^{2}\left(\frac{x^{3}}{x_{0}^{3}}\right)}\right]^{\prime \prime}\right|_{x=x_{0}}\right\}, \\
\lambda^{(2)}=-\frac{4 \pi^{2}}{3} \frac{k^{2} T n_{e}}{m_{e}} \frac{\tau_{d}^{2} \omega}{B}\left\{\frac{1}{1+\omega^{2} \tau_{d}^{2}}-\frac{3}{4} \frac{\omega^{2} \tau_{d}^{2}}{\left(1+\omega^{2} \tau_{d}^{2}\right)^{2}}-\left.\frac{\pi^{2}}{16}\left[\frac{1}{1+\omega^{2} \tau_{d}^{2}\left(\frac{x^{3}}{x_{0}^{3}}\right)}\right]^{\prime \prime}\right|_{x=x_{0}}\right\} . \\
B^{2} \lambda^{(3)}=\lambda^{(1)}(B=0)-\lambda^{(1)} .
\end{gathered}
$$

In the case of strongly degenerate electrons the equations (3.9)-(3.11),(3.18),(3.19) give an asymptotically exact solution for the heat conductivity coefficients, because collisions between electrons can be neglected in this case. The difference between the exact $\left[\lambda^{(1)}\right] /\left[\lambda^{(1)}(B=0)\right]$ from (3.18), and phenomenological account of the magnetic field influence on the heat conductivity coefficients (1.1) is presented in Fig. 1. Here the ratios between the values, which are perpendicular and parallel to magnetic field, are plotted for $k T=0.09 E_{f}$. At $\omega \tau=1.5$ the exact value of this ratio is 4 times smaller than the phenomenological one. 


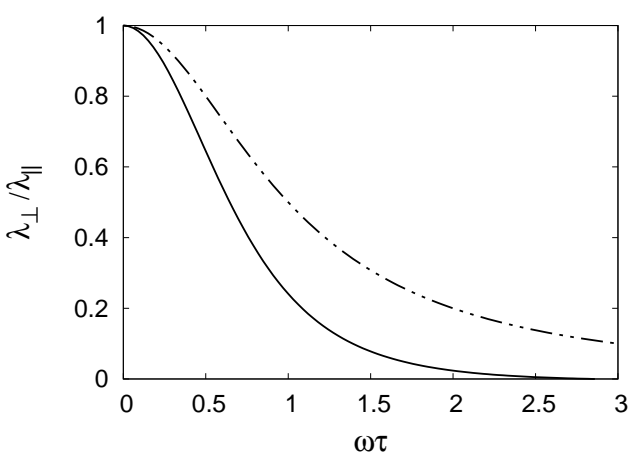

Figure 3: The plots of the ratio $\lambda_{\perp} / \lambda_{\|}$as a function of $\omega \tau$ are presented for phenomenologically obtained heat conductivity (dash-dot line) for comparison with heat conductivity obtained by the solution of Boltzmann equation in Lorentz appoximation (solid line) with $k T=0.09 E_{f}$.

\section{Discussion}

In this paper a thermal conductivity tensor is found for degenerate non-relativistic electrons in presence of a non-quantizing magnetic field. The heat conductivity coefficients for strongly degenerate electrons, in presence of magnetic field, are obtained asymptotically exactly in Lorentz approximation, when the electron-electron collision may be neglected in comparison with electronnuclei collisions at nondegenerate nuclei.

In most works considering the heat conductivity in astrophysical objects, in the neutron stars in particular, following Flowers and Itoh [8], the influence of the magnetic field on the heat flux was taken into account phenomenologically using the coefficient $1 /\left(1+\omega^{2} \tau^{2}\right)$, which decreases the heat flux in the direction perpendicular to the direction of a magnetic field. Our results, obtained by the solution of Boltzmann equation show, that the influence of the magnetic field on the coefficients of heat conductivity is stronger, and has a more complicated character Fig. 1.

The new coefficients can be used for calculation of temperature distribution in white dwarfs, near the surface, and in the crust of magnetized neutron star. The temperature distribution over the surface of NS is important for understanding of the geometry of magnetic field inside the neutron star and near its surface.

\section{Acknowledgments}

This work was supported by a grant from RFBR (project No. 17-02-00760).

\section{References}

[1] Bisnovatyi-Kogan G.S., Journal of Applied Mechanics and Technical Physics 3 (1964) 43.

[2] Bisnovatyi-Kogan G.S., Transport properties of partially ionized two-temperature plasma and isotropic corrections to maxvellian distribution function (in Russian), Diploma thesis, Moscow Institute of Physics and Technology (1964).

[3] Bisnovatyi-Kogan G.S., Glushichina M.V., Plasma Physics Reports 44 (2018) No.4; arXiv:1606.05226v2. 
[4] Bisnovatyi-Kogan G.S., Kulikov Yu.N., Chechetkin V.M., Sov. Astron. 20 (1976) 552.

[5] Bisnovatyi-Kogan G.S., Romanova M.M., JETP 56 (1983) 243.

[6] Burnett D., Proceedings of London Mathematical Society40 (1936) 382.

[7] Chapmen S., Cowling T.G., Mathematical Theory of Nonuniform Gases, (Cambrige, 1952) [Russian translation published by IL, Moscow, 1958].

[8] Flowers E., Itoh N., Astrophysical Journal 206 (1976) 218.

[9] Ginzburg V.L., Rukhadze A.A., Waves in Magnetoactive Plasma, (Nauka, Moscow, 1970).

[10] Haberl F., Schwope A.D., Hambaryan V., Hasinger G., and Motch C., Astron. Astrophys 403 (2003) L19.

[11] Itoh N., Hayashi H., Kohyama Y., Astrophysical Journal 418 (1993) 405.

[12] Lampe M., Physical Review 170 (1968) 306.

[13] Landau L.D., Lifshitz E.M., Statistical Physics. 3rd ed., (Butterworth-Heinemann, 1980).

[14] Landshoff R., Physical Review 82 (1951) 442.

[15] Marshall W., The kinetic theory of an ionized gas. p. 3 At. Energy Res Estable, N T/R, 2419, (1960).

[16] Salpeter E.E., Astrophysical Journal 134 (1961) 669.

[17] Tomonaga S.Z., Z. Phys. 110 (1938) 573.

[18] Urpin V.A., Yakovlev D.G., Astron. Zh. 57 (1980) 526.

[19] Wikipedia: https ://en.wikipedia.org/wiki/The_Magnificent_Seven_(neutron_stars).

[20] Wyller A.A., Astrophisica Norvegica 9 (1964) 79.

[21] Yakovlev D.G., Gnedin O.Y., Kaminker A.D., Levenfish K.P., Potekhin A.Y., Advances in Space Research 33 (2006) 523.

[22] Yakovlev D.G., Levenfish K.P., Shibanov Yu.A., Uspekhi Fiz. Nauk 169 (1999) 825.

[23] Zavlin V.E., Astrophysical Journal 665 (2007) L143. 